Mots. Les langages du politique

Discours d'Amérique latine. Identités et conflits

\title{
Angenot, M. (2014). L'Histoire des idées
}

Liège : Presses universitaires de Liège

\section{Chloé Gaboriaux}

\section{(2) OpenEdition}

\section{Journals}

Édition électronique

URL : https://journals.openedition.org/mots/22173

DOI : $10.4000 /$ mots.22173

ISSN : 1960-6001

Éditeur

ENS Éditions

\section{Édition imprimée}

Date de publication : 30 novembre 2015

Pagination : 151

ISBN : 978-2-84788-776-1

ISSN : 0243-6450

\section{Référence électronique}

Chloé Gaboriaux, «Angenot, M. (2014). L'Histoire des idées », Mots. Les langages du politique [En ligne], 109 | 2015, mis en ligne le 30 novembre 2015, consulté le 23 avril 2022. URL : http:// journals.openedition.org/mots/22173; DOl : https://doi.org/10.4000/mots.22173 


\title{
Compte rendu de lecture
}

\author{
L'Histoire des idées \\ Marc Angenot \\ 2014, Liège, Presses universitaires de Liège, 392 pages
}

Avec son dernier livre, Marc Angenot s'aventure sur un terrain miné, du moins dans l'univers académique francophone. En effet, si l'histoire des idées a pignon sur rue dans de nombreux pays, au Royaume-Uni ou en Allemagne par exemple, elle suscite ici la réticence. Dans certains domaines, elle en est même venue à incarner tout ce contre quoi le chercheur en sciences sociales doit se prémunir: c'est l'histoire de l'idée $X$ ou Y de Platon à nos jours, un récit à la fois nominaliste et téléologique, indifférent aux conditions d'énonciation et de production des discours et des croyances et naïvement imprégné des préjugés de son auteur, bref, anachronique à tous égards.

Pourtant, souvent dissimulé sous d'autres appellations, l'intérêt pour les idées ne s'est jamais démenti et, s'il a pris des formes variées et parfois contradictoires, il ne s'est jamais non plus confondu avec son stéréotype négatif. C'est l'objectif premier de la démonstration de Marc Angenot dans L'Histoire des idées : rendre justice à ces travaux sans acrimonie mais sans concession, «montrer les difficultés inhérentes, les présupposés contradictoires, les limites, en même temps que le côté fructueux, l'intérêt, la portée de certaines démarches et certaines problématiques» (p. 22).

Il en résulte un ouvrage dense, à la fois déconcertant et stimulant. La forme, d'emblée, peut surprendre : il s'agit d' " une sorte de gros essai » (p. 5), qui mêle observations épistémologiques et commentaires plus personnels, exemples empiriques et interrogations réflexives. La lecture ressemble à une discussion vive et serrée, dont la complexité est à la mesure de son ouverture intellectuelle : les lecteurs de l'œuvre de Marc Angenot retrouveront dans L'Histoire des idées son érudition œcuménique et généreuse, retenant ici ou là ce qui est bon à prendre, au risque de perdre les esprits pusillanimes en attente de vérités tranchées et de jugements manichéens.

L'auteur applique en effet à son travail les règles de méthode qu'il préconise, réalisant dès lors trois ouvrages en un. L'Histoire des idées est bien sûr avant tout, ce qu'elle annonce en début de parcours, une histoire des histoires des idées, construite autour des grands enjeux soulevés par ce type 
d'approches. Après avoir évoqué leur peu de visibilité et de reconnaissance en France, qui contraste avec leur institutionnalisation ailleurs («chapitre premier : une discipline illégitime »), l'auteur consacre quatre chapitres à la façon dont elles se positionnent vis-à-vis de la dimension collective des idées - qui implique de renoncer au mythe du grand auteur génial mais pose néanmoins un certain nombre de questions épistémologiques (chapitre II: «Des idées collectives ») -, des objets à privilégier (chapitre III : «Une diversité d'objets »), du rôle de l'historien des idées (chapitre IV : «Les représentations du passé »), et de la causalité et plus largement du rapport établi entre les idées, d'une part, et les faits matériels et les pratiques concrètes, d'autre part (chapitre V : «Origines intellectuelles des événements historiques. Le rôle des idées dans l'histoire et leurs "responsabilités" »).

Parce qu'elle se nourrit d'exemples précis, cette histoire des histoires des idées suggère aussi ce que pourrait être une histoire de la pensée contemporaine depuis le XVIII siècle, de ses schèmes structurants et de ses apories. Et comme elle questionne sans cesse l'historicité de ses propres catégories d'analyse, on peut y suivre un troisième fil directeur, mettant en abyme de façon un peu vertigineuse la double entreprise menée simultanément sur le front des différentes démarches en histoire des idées et sur celui des terrains empiriques examinés... L'ouvrage revient ainsi pour finir sur les critiques adressées à l'histoire des idées (chapitre $\mathrm{VI}:$ «Objections faites à l'histoire des idées »), poury répondre par des recommandations méthodologiques qui ont par ailleurs été mises en œuvre, explicitement ou non, tout au long de l'ouvrage (chapitre VII : «Règles heuristiques. Ethos recommandé à l'historien des idées»).

De ce livre foisonnant, on ne présentera ici que quelques-uns des apports, ceux qui nous ont paru le mieux à même de répondre aux objections généralement formulées contre l'histoire des idées. Le premier tient sans doute à la façon dont Marc Angenot désamorce les exigences exorbitantes que la liste infinie des objections faites à l'histoire des idées fait paradoxalement peser sur elle. Il s'agit en effet d'abord d'écarter celles qui reviennent in fine à reprocher à l'histoire des idées de focaliser son attention sur... les idées. Comme le rappelle Marc Angenot, «toute historiographie est sectorielle, elle isole un segment d'un tout hétérogène trop complexe pour être maîtrisable» (p. 307). Autrement dit, l'étude des idées est tout aussi légitime que celle d'autres aspects de la réalité, à condition qu'elle obéisse aux critères de scientificité du moment.

Cette sage mise en garde lui permet en outre de dépasser l'opposition artificielle des idées et des pratiques : "Les relations sociales n'existent et ne se spécifient qu'à travers des valeurs, des conceptions et des représentations, il est donc permis d'isoler celles-ci pour fins d'analyse - alors que se demander indéfiniment ce qui est ultimement “déterminant”, c'est ressasser le vain dilemme paralogique de l'œuf et de la poule » (p.310). Qu'on les appelle valeurs, croyances, normes, etc., les idées sont indissociables des pratiques 
qu'elles rendent socialement signifiantes et dont elles se nourrissent tout à la fois, comme délimitations du pensable et du faisable, institutions du sens ou rationalisations a posteriori.

Dans cette perspective, le souci d' " isoler les idées» ne revient pas à les couper du réel dont elles sont de toute façon partie prenante mais à en faire une entrée privilégiée dans la compréhension de la société. C'est pourquoi Marc Angenot envisage difficilement une histoire des idées qui ne serait pas en même temps une histoire des discours, quelles qu'en soient les méthodes. Comment saisir l'historicité des idées, sinon dans la façon dont elles ont été exprimées à un moment donné par des locuteurs donnés? Comment appréhender l'inscription des idées dans la société, sinon à travers des énoncés particuliers, qui mêlent inextricablement manières de dire héritées et reformulations singulières? D’où cette injonction exigeante mais incontournable : «Un historien des idées doit avoir, il doit s'être donné pour pratiquer son métier une formation tant en linguistique et en pragmatique qu'en analyse du discours (ce qui inclut la rhétorique argumentative, la logique informelle et la narratologie)» (p.157).

À cet égard, on regrettera que l'auteur néglige une question pourtant souvent adressée à l'histoire des idées : celle de la «représentativité » des idées étudiées et, partant, des énoncés sélectionnés. Sans doute paraissait-elle évidente à un chercheur connu pour avoir travaillé sur des corpus ambitieux, qui lui donnent une exceptionnelle maîtrise des enjeux relatifs au « discours social » des périodes qu'il étudie. C'est cette dernière qui le conduit à affirmer son bonheur «quand il rencontre dans un écrit la formulation nucléaire d'un discours dans sa parfaite spécificité et sa parfaite évidence» (p.186). Il est en effet des locuteurs qui, au détour d'une phrase, parviennent à condenser en une expression fulgurante l'articulation logique des raisonnements largement partagés et diversement déclinés par leurs contemporains. Mais seule une connaissance approfondie de ce qui s'écrit et se dit à un moment donné permet à l'historien de repérer ces énoncés rares et de s'en réjouir. Dans le cas contraire, il risque fort d'investir un texte isolé de ses propres préoccupations et de généraliser abusivement le cas individuel au reste de la société.

Suivre l'appel à la réflexivité sous-jacent à tout le livre de Marc Angenot devrait cependant l'en préserver. Regrettant le malentendu suscité en France par le linguistic turn, l'auteur ne cesse en effet d'inviter l'historien des idées à procéder à l'analyse de son propre discours en même temps qu'il examine ceux des locuteurs du passé. C'est toute la beauté et la difficulté de l'entreprise, que Marc Angenot évoque dans un épilogue empreint du doute propre aux grands chercheurs : "Comment faire un travail dont les outils, évanescents, incertains et controuvés, sont identiques au matériau?» (p. 355).

Chloé Gaboriaux

IEP Lyon, Triangle (UMR 5206 du CNRS) 